\title{
Cyclic Fatigue of Glide Path Rotary NiTi Files in a Double (S-Shaped) Simulated Curvature
}

\section{Dina Al-Sudani ${ }^{1 *}$, Gianluca Plotino ${ }^{2}$, Nicola M Grande ${ }^{2}$, Sandro Rengo ${ }^{3}$, Michele Simeone ${ }^{3}$ and Gianluca Gambarini ${ }^{2}$}

${ }^{1}$ Department of Restorative Dental Sciences, College of Dentistry, King Saud University, Riyadh, Saudi Arabia

${ }^{2}$ Department of Oral Science, "Sapienza" University of Rome, Rome, Italy

${ }^{3}$ Department of Oral and Maxillofacial Sciences, University of Naples "Federico II", Napoli, Italy

\begin{abstract}
Objective: The aim of the present study was to compare the fatigue resistance of PathFile (PF) and ProGlider (PG) $\mathrm{NiTi}$ (Nickel-Titanium) rotary files in a double (S-shaped) curvature artificial root canal.

Methods: The cyclic fatigue of the following rotary NiTi glide path instruments was tested in a double curvature artificial canal, PF (tip size .16 and .02 taper) and PG (tip size .16 and variable taper). Twenty instruments for each group were tested to fracture in continuous rotary motion at $300 \mathrm{rpm}$. The number of cycles to failure (NCF) was calculated and the length of the fractured fragment was measured. Data were statistically analyzed with a level of significance set at $5 \%$.

Results: There was no significant difference in cyclic fatigue resistance between the PF and the PG in the apical curvature $(p>0.05)$. However, in the coronal curvature the NCF value was significantly higher for the PG than for the PF $(p<0.05)$. The NCF values were significantly lower $(p<0.05)$ in the apical curvature of the artificial canal than in the coronal curvature for both instruments. No differences in the length of the fractured fragments were found $(p>0.05)$.

Conclusion: The instruments were found to be less resistant to cyclic fatigue in the apical curvature of the artificial canal than in the coronal curvature. PG instrument showed significantly greater cyclic fatigue resistance in the coronal curvature.
\end{abstract}

Keywords: NiTi; Cyclic fatigue; Glide path; ProGlider; PathFile

\section{Introduction}

One of the main goals of the manufacturers of Nickel-Titanium (NiTi) rotary instruments is to reduce the likelihood of instrument separation and improve safety through innovative design and manufacturing processes [1,2]. Cyclic fatigue and torsional fracture are the two main mechanisms that may lead to instrument separation, mainly caused by bending and torsional stress [3]. Canal curvature is the predominant risk factor for increased bending stress and a clinician may have no influence on this parameter [4]. One of the recommendations to reduce fracture risk of $\mathrm{NiTi}$ instruments is to create a glide path during the initial preparation [5]. The endodontic glide path, which has been described as having sufficient patency from the canal orifice to the apical foramen, is performed using smallsized and slightly tapered NiTi rotary instruments or stainless-steel manual files [5]. The creation of a glide path may facilitate root canal preparation when $\mathrm{NiTi}$ instruments with larger tapers are used and reduce the incidence of procedural errors $[6,7]$.

Recently, considerable focus was given to NiTi rotary instruments designed for glide path preparation. PathFile (Dentsply-Maillefer, Ballaigues, Switzerland) is an endodontic rotary pathfinding system consisting of 3 instruments of different tip size and same .02 constant taper manufactured from conventional $\mathrm{NiTi}$ with a four cutting edges square cross-section [7]. ProGlider (Dentsply-Maillefer, Ballaigues, Switzerland) is a novel single-file rotary pathfinding instrument manufactured from heat-treated $\mathrm{M}$-wire alloy with a progressive taper from $2 \%$ to $8 \%$ over its length and a four cutting edges square crosssection [7].

The fracture resistance of pathfinding instruments is just as important as that of instruments used to shape the root canals [8]. Consequently, endodontic instruments used for the negotiation of narrow and curved root canals should have mechanical properties that allow for safe apical progression and efficient performance [9-11]. The aim of the present study was to compare the cyclic fatigue resistance of PathFile (PF) and ProGlider (PG) NiTi rotary glide path files in a double (S-shaped) curvature artificial root canal.

\section{Materials and Methods}

The cyclic fatigue resistance of the following rotary NiTi pathfinding instruments was tested in a double curved artificial canal, PF (tip size .16 and .02 taper) and PG (tip size .16 with a variable taper). Instruments were tested for fatigue in a specific cyclic fatigue device that specifically developed and it has been used in previous studies [1-5,12]. The PF and PG instruments were then subjected to cyclic fatigue test using a mechanical device (Figure 1). The apparatus was connected to an endodontic motor and enabled the instrument to rotate freely within a stainless steel artificial canal at a constant pressure. The artificial canal was manufactured by reproducing an instrument's size and taper. It provided the instrument with a suitable simulated root canal with a double (S-shaped) curvature. The first curvature was a coronal curve that had a $60^{\circ}$ angle of curvature and a radius of $5 \mathrm{~mm}$, and it was located $6 \mathrm{~mm}$ from the tip of the instrument. The second curvature was an apical curve that had a $70^{\circ}$ angle of curvature and a radius of $2 \mathrm{~mm}$, and it was located $2 \mathrm{~mm}$ from the tip.

*Corresponding author: Dina Al-Sudani, Professor of Endodontics Department of Restorative Dental Sciences, College of Dentistry, King Saud University, P.O. Box 60169, Riyadh 11545, Saudi Arabia, Tel: +96 6504441941; E-mail: dinaalsudani@gmail.com

Received December 21, 2015; Accepted January 06, 2015; Published January 13, 2016

Citation: Al-Sudani D, Plotino G, Grande NM, Rengo S, Simeone M, et al. (2016) Cyclic Fatigue of Glide Path Rotary NiTi Files in a Double (S-Shaped) Simulated Curvature. Dentistry 6: 355. doi:10.4172/2161-1122.1000355

Copyright: (c) 2016 Al-Sudani D, et al. This is an open-access article distributed under the terms of the Creative Commons Attribution License, which permits unrestricted use, distribution, and reproduction in any medium, provided the original author and source are credited. 


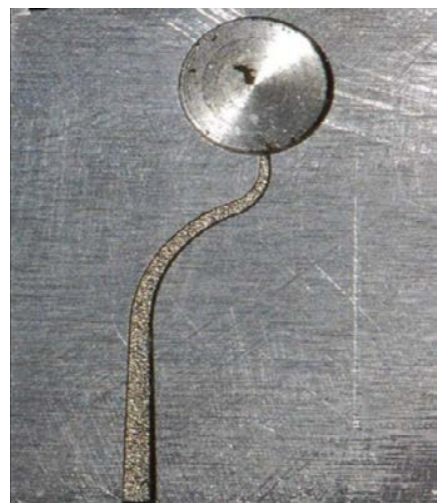

Figure 1: Artificial double curvature root canal used to test cyclic fatigue.

\begin{tabular}{|c|c|c|c|c|}
\hline \multirow{2}{*}{ Instrument size } & \multicolumn{4}{|c|}{ Double Curvature } \\
\cline { 2 - 5 } & \multicolumn{2}{|c|}{ Apical curve } & \multicolumn{2}{c|}{ Coronal curve } \\
\cline { 2 - 5 } & NCF (SD) & FL (SD) & NCF (SD) & FL (SD) \\
\hline ProGlider 16/.04 & $231.1(32.9)$ & $1.6(0.25)$ & $1120(49.9)$ & $4.3(0.25)$ \\
\hline PathFile 16/.02 & $252.5(82.9)$ & $1.7(0.25)$ & $696(104.1)$ & $4.6(0.21)$ \\
\hline
\end{tabular}

Table 1: Mean (SD) of NCF and Fragment Length (FL) in $\mathrm{mm}$ registered during Cyclic Fatigue Testing in the Double (S-shaped) curvature.

Twenty instruments were rotated at a constant speed of $300 \mathrm{rpm}$ using a 6:1 reduction hand piece (Sirona Dental Systems GmbH, Bensheim, Germany) powered by a torque-controlled electric motor (VDW Silver, VDW GmbH-Dentsply International Inc, Munich, Germany). Torque was set at $2 \mathrm{~N} / \mathrm{cm}$. To reduce the friction of the file as it contacted the artificial canal walls, special high-flow synthetic oil was designed for the lubrication of mechanical parts (Super Oil; Singer Co Ltd, Elizabethport, NJ). For each instrument, the time in seconds from the start of the test until the moment of breakage was recorded with a chronometer to an accuracy of 0.1 seconds, and the number of cycles to failure (NCF) were calculated to the nearest full number multiplying the seconds by 5 (number of cycles for second using $300 \mathrm{rpm}$ ). Mean values and standard deviations (SD) were then calculated for each group. Cyclic fatigue data were analyzed by one-way analysis of variance and Tukey's HSD test to determine any significant differences between the groups, and the level of significance was set at $5 \%$. Additionally, Student's t-test was performed to compare the fragment lengths between different groups.

\section{Results}

Mean values \pm SD expressed as NCF are displayed in (Table 1). A higher NFC was correlated with a higher resistance to cyclic fatigue of the tested instruments. In the double curvature model, there was no significant difference in cyclic fatigue between the PF and the PG in the apical curvature. However, the NCF was significantly higher in the coronal curvature for the PG than for the PF $(p<0.05)$. The NCF value was significantly lower in the apical curvature than in the coronal curvature in both of the instruments $(\mathrm{p}<0.05)$. Furthermore, for all instruments there were no statistically significant differences in the mean length of fractured segments $(\mathrm{p}<0.05)$.

\section{Discussion}

The endodontic glide path is defined as a smooth radicular tunnel from the canal orifice of the canal to the physiologic terminus of the root canal [13]. Glide path is achieved when the file creating it, can enter from the orifice and follow the smooth canal walls uninterrupted to the terminus [13].

This initial root canal preparation is usually performed before the intracanal use of any NiTi rotary instrument to reduce the effect of torsional stress along the canal and risk of instrument failure. In fact, the risk of instrument fracture is still a concern, particularly in the use of small instrument files for glide path preparation.

Achieving patency is considered a clinical challenge during the treatment of a double (S-shaped) curvature root canal, whose frequency is not rare [14]. In the present study, the fatigue resistance of PF and PG instruments was compared in a double (S-shaped) curvature artificial root canal. Comparison of the coronal and apical portions of the double curvature showed that both instruments were less resistant to cyclic fatigue in the apical portion of the canal. A possible explanation is that the apical curvature was more abrupt, with a radius of only 2 $\mathrm{mm}$, than the coronal curvature, which had a $5 \mathrm{~mm}$ radius. This result is in agreement with previous studies, which demonstrated that the fatigue life of NiTi rotary instruments is significantly influenced by the angle and radius of the curvature $[4,7,12,15]$.

The results of the present study showed that there was no significant difference between PG and PF instruments in the most complex apical curvature. However, PG instruments had significantly greater resistance to cyclic fatigue in the coronal curvature than $\mathrm{PF}$, despite its progressive taper, which is from $2 \%$ to $8 \%$ over its length. Alternatively, the fixed .02 taper of the PF file results in an instrument with a smaller metal core that usually leads to an enhanced cyclic fatigue resistance [4]. This could be attributed to the different manufacturing processes used for the instruments tested, thus confirming the results of several previous studies that compared M-Wire NiTi instruments with traditional NiTi rotary instruments and found that M-Wire improves resistance to fracture caused by cyclic fatigue stress [7,10,16-18]. In fact, these studies showed that the fatigue life of rotary instruments is extremely sensitive to the raw materials used in the manufacturing process. A study, reported that PG NiTi pathfinding instruments made of M-Wire alloy had enhanced mechanical properties, including higher flexibility and higher resistance to cyclic fatigue and torsional stress, than PF instrument made of conventional NiTi alloy $[7,19]$. However, the PF instrument manufactured from conventional NiTi also showed superior cyclic fatigue resistance in the apical curvature, this result is in accordance with the recent study by Capar et al. in which they attributed their results to the instrument design [7]. In the present study, despite the different manufacturing processes used for the instruments, both instruments have a square cross section. Under the experimental conditions and the limitations of the present study, the instruments were found to be less resistant to cyclic fatigue in the apical curvature of the double (S-shaped) artificial canal than in the coronal curvature. PG instrument showed significantly greater cyclic fatigue resistance than PF in the coronal curvature, while no significant difference was found in the apical curvature.

\section{Conclusion}

The results of the present study showed that there was no significant difference between PG and PF instruments in the most complex apical curvature. The instruments were found to be less resistant to cyclic fatigue in the apical curvature of the artificial canal than in the coronal curvature. PG instrument showed significantly greater cyclic fatigue resistance in the coronal curvature. 
Citation: Al-Sudani D, Plotino G, Grande NM, Rengo S, Simeone M, et al. (2016) Cyclic Fatigue of Glide Path Rotary NiTi Files in a Double (S-Shaped) Simulated Curvature. Dentistry 6: 355. doi:10.4172/2161-1122.1000355

\section{References}

1. Plotino G, Grande NM, Cotti E, Testarelli L, Gambarini G (2014) Blue treatment enhances cyclic fatigue resistance of vortex nickel-titanium rotary files. J Endod 40: 1451-1453

2. Pedullà E, Grande NM, Plotino G, Gambarini G, Rapisarda E (2013) Influence of continuous or reciprocating motion on cyclic fatigue resistance of 4 different nickel-titanium rotary instruments. J Endod 39: 258-261.

3. Plotino G, Grande NM, Cordaro M, Testarelli L, Gambarini G (2009) A review of cyclic fatigue testing of nickel-titanium rotary instruments. J Endod 35: 14691476.

4. Grande NM, Plotino G, Pecci R, Bedini R, Malagnino WA, et al. (2006) Cyclic fatigue resistance and three-dimensional analysis of instruments from two nickel-titanium rotary systems. Int Endod J 39: 755-763.

5. Gambarini G, Plotino G, Sannino G, Grande NM, Giansiracusa A, et al. (2015) Cyclic fatigue of instruments for endodontic glide path. Odontology 103: 56-60.

6. Ha JH, Park SS (2012) Influence of glide path on the screw-in effect and torque of nickel titanium rotary files in simulated resin root canals. Restor Dent Endod 37: 215-219.

7. Capar ID, Kaval ME, Ertas H, Sen BH (2014) Comparison of the cyclic fatigue resistance of 5 different rotary pathfinding instruments made of conventional nickel-titanium wire, M-wire, and controlled memory wire. J Endod 41: 535-538.

8. Sung SY, Ha JH, Kwak SW, Abed RE, Byeon K, et al. (2014) Torsional and cyclic fatigue resistances of glide path preparation instruments: G-file and PathFile. Scanning 36: 500-506.

9. Allen MJ, Glickman GN, Griggs JA (2007) Comparative analysis of endodontic pathfinders. J Endod 33: 723-726.
10. Lopes HP, Elias CN, Amaral G, Vieira VT, Moreira EJ, et al. (2011) Torsional properties of pathfinding instruments. Oral Surg Oral Med Oral Pathol Oral Radiol Endod 112: 667-670.

11. Lopes HP, Elias CN, Siqueira JF Jr, Soares RG, Souza LC, et al. (2012) Mechanical behavior of pathfinding endodontic instruments. J Endod 38: 14171421.

12. Al-Sudani D, Grande NM, Plotino G, Pompa G, Di Carlo S, et al. (2012) Cyclic fatigue of nickel-titanium rotary instruments in a double (S-shaped) simulated curvature. J Endod 38: 987-989.

13. West J (2006) Endodontic update 2006. J Esthet Restor Dent 18: 280-300.

14. Saleh AM, Vakili Gilani P, Tavanafar S, Schäfer E (2015) Shaping ability of 4 different single file systems in simulated S-shaped canals. J Endod 41: 548552.

15. Pruett JP, Clement DJ, Carnes DL Jr. (1997) Cyclic fatigue testing of nickeltitanium endodontic systems. J Endod 23: 77-85

16. Alapati SB, Brantley WA, lijima M, Clark WAT, Kovarik L, et al. (2009) Metallurgical characterization of a new nickel titanium wire for rotary endodontic instruments. J Endod 35: 1589-1593.

17. Gao Y, Shotton V, Wilkinson K, Phillips G, Johnson WB (2010) Effects of raw material and rotational speed on the cyclic fatigue of ProFile Vortex rotary instruments. J Endod 36: 1205-1209.

18. Capar ID, Ertas H, Arslan H (2014) Comparison of cyclic fatigue resistance of nickel-titanium coronal flaring instruments. J Endod 40: 1182-1185.

19. Elnaghy AM, Elsaka SE (2014) Evaluation of root canal transportation centering ratio, and remaining dentin thickness associated with ProTaper Next instruments with and without Glide Path. J Endod 40: 2053-2056. 